\title{
APPARENT FREE AMINO ACIDS IN DEPROTEINIZED PLASMA OF NORMAL AND UREMIC PERSONS ${ }^{1}$
}

\author{
By PETER F. SALISBURY, MAX S. DUNN, AND EDWARD A. MURPHY \\ (From the Institute for Medical Research, Cedars of Lebanon Hospital, and the Chemical \\ Laboratory, University of California, Los Angeles, Calif.)
}

(Submitted for publication February 11, 1957; accepted April 4, 1957)

Not all clinical manifestations of severe uremia can be explained on the basis of abnormalities of the water balance or of electrolyte patterns. Many patients die with the uremic syndrome even as the plasma levels of all known electrolytes are normal $(1,2)$. Even though it appears unlikely that retention of the known end products of protein metabolism is responsible for the clinical picture of uremia (3), it is probable that the toxicity of the uremic syndrome is related to a disturbance of protein metabolism. Measurement and comparison of free amino acid concentrations in the body fluids of uremic persons with the amino acid patterns in normal body fluids is prerequisite to further investigations of protein metabolism in uremia. If one or more of the essential amino acids were found deficient in uremic body fluids such a finding might serve to explain certain clinical features of the uremic syndrome which suggest decreased protein synthesis. Abnormally high concentrations of individual amino acids in uremic body fluids would raise the question of their contribution to the uremic toxicity. Consistently abnormal amino acid patterns in uremia would call for explorations of the mechanisms underlying this abnormality. Such investigations might fill in and define our presently so vacuous and chimerical picture of the derangements of chemical and physiological processes which are the basis for the uremic syndrome.

Animal experiments from our laboratories (4) have demonstrated deficiencies of apparent free methionine and threonine, with simultaneous excess of apparent free arginine and glycine in the blood plasma of nephrectomized uremic dogs. The present report extends our studies of plasma amino acid levels in uremia to human patients.

1 Supported in part by grants from the U. S. Public Health Service, the American Cancer Society, and the University of California.

\section{METHODS}

Plasma was obtained from six human patients with severe uremia caused by chronic glomerular nephritis. The procurement of the plasma specimens coincided with replacement transfusions which had become indicated in these patients because of severe signs and symptoms of uremia and because of the coexisting anemia. Since the blood specimens were withdrawn from the patients simultaneously with the administration of identical volumes of citrated blood, they became diluted with donor blood during withdrawal. It is, however, estimated that none of the uremic specimens contain more than 25 per cent normal donor blood because only the first $1000 \mathrm{ml}$. of the withdrawn blood were used for amino acid assay. The dilution factor would tend to diminish any differences which exist between uremic blood and bank blood, and the abnormalities actually existing in uremic blood must be more pronounced than our data would indicate. Aside from the replacement transfusions, the patients had the therapeutic regimen customary in uremic patients : water and electrolyte balance, a high-caloric, lowprotein diet, and vitamin supplements. Three patients were digitalized, three received daily injections of testosterone propionate, one (E. H., No. 4) had had a course of antibiotics one week before the procurement of the plasma specimen (antibiotics may interfere with microbiological amino acid assay). Plasma samples were taken only from patients with chronic uremia due to primary renal disease because it was felt that this would reduce the variability of blood amino acid levels which might be introduced by the coexistence of uremia with debridement and digestion of necrotic tissues (as in acute renal failure or in simultaneous severe traumatic injuries).

Five hundred $\mathrm{ml}$. blood samples were also taken from nine healthy young men who had been without food for ten hours.

All blood was taken from the venous side of the circulation and collected in siliconed glass bottles which contained $30 \mathrm{mg}$. heparin per $500 \mathrm{ml}$. blood. The heparinized blood was transferred to the cold room where it was allowed to settle by gravity. One or two days after collection the supernatant plasma was syphoned off, collected in siliconed glass bottles and frozen. It was preserved in the frozen state up to the time of further processing.

Deproteinization was effected by adding per $\mathrm{ml}$. of plasma $1.5 \mathrm{ml}$. of approximtaely 4 per cent sodium tung- 
state in $0.35 \mathrm{~N} \mathrm{H}_{2} \mathrm{SO}_{4}$. The mixture was allowed to stand for 30 minutes with occasional shaking, the suspension was filtered on a Buchner funnel, the residue was washed twice with a minimum of distilled water, and the washings were added to the filtrate. The filtrate was evaporated to dryness under reduced pressure to residual material which was taken up in warm distilled water and, after filtration, aliquots were diluted with distilled water to a volume such that $1 \mathrm{ml}$. was equivalent to approximately 0.5 and $1.5 \mathrm{ml}$. of the original plasma, respectively.

For purposes of hydrolysis of amino acid conjugates an aliquot of the condensed filtrate of one sample was made $6 \mathrm{~N}$ in acid with $\mathrm{HCl}$, refluxed for 24 hours, filtered, the residue washed with a minimum of warm water, and the washings added to the filtrate. This filtrate was then treated in the same manner as that described above.

The assay procedures have been described previously $(5,6)$.

\section{RESULTS AND DISCUSSION}

\section{Concentration of apparent free amino acids in normal human plasma}

It is evident from Table I that the authors' values for apparent free amino acids in normal plasma are comparable to values obtained elsewhere both microbiologically and by column chromatography for aspartic acid, glutamic acid, methionine, phenlalanine, serine and valine. Our values for arginine, histidine and proline were lower than concentrations observed elsewhere. Values obtained here for glycine and threonine were higher than those determined with chromatography elsewhere, and our measurements of the concentrations of isoleucine, leucine and lysine were lower

TABLE I

Concentration of apparent free amino acids in normal human plasma

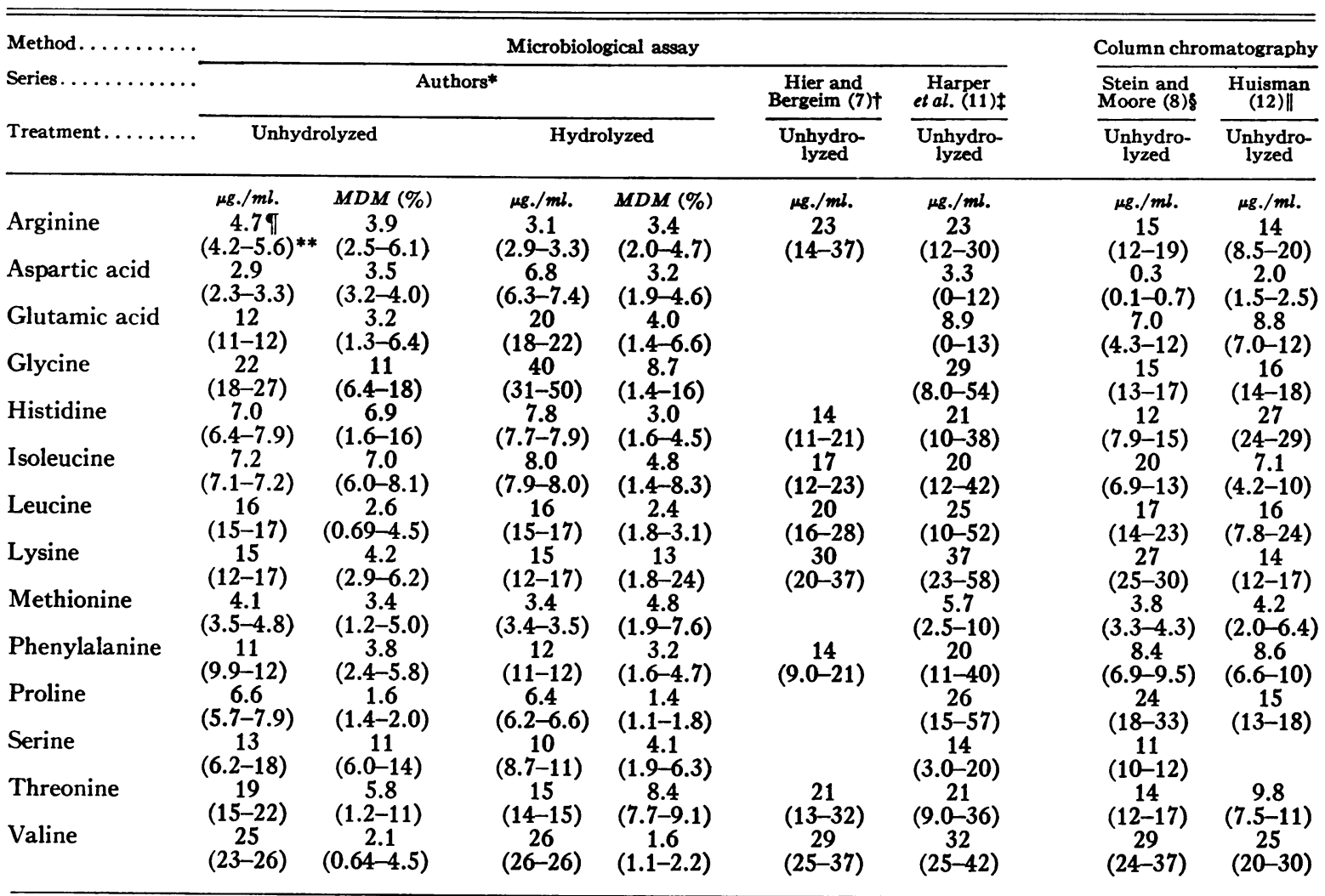

* Pooled sample from nine normal men, fasted. Average of 2 to 3 assays at 2 to 3 different sample concentrations (0.45 to 1.5 relative to fresh plasma).

t Average value from 34 normal men, fasted.

† Average value from 17 normal men, fasted.

\& Average value from five normal men, fasted.

Average value from two normal men, three hours after feeding.

it Average value.

** Range of values. 
TABLE II

Concentration of apparent free amino acids in uremic human plasma from one patient $(G$.$) and its$ relationship to that found in normal human plasma

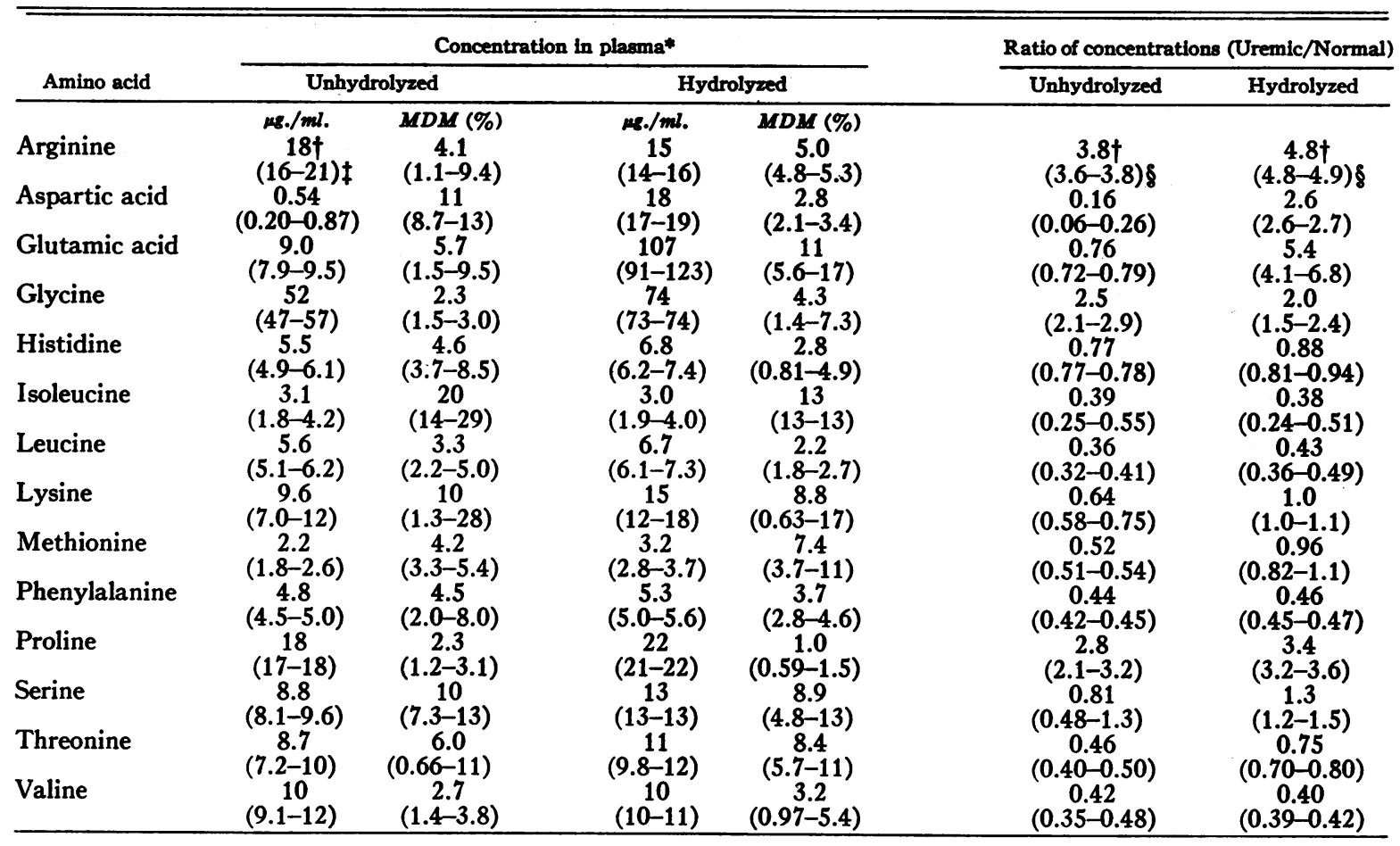

* Pooled samples from a single uremic patient.

t Average values from 2 to 3 assays at 2 to 3 different sample concentrations ( 0.40 to 1.5 relative to fresh plasma). $\mp$ Range of values.

Range of values of ratios at different but equal plasma sample concentrations.

than data obtained elsewhere with microbiological methods.

It should be noted that there was no change in the concentration of histidine, lysine, phenylalanine, proline and valine before and after complete hydrolysis of the plasma. The mean deviation from the means observed at twelve levels of sample ranging from 0.08 to $1.2 \mathrm{ml}$. of plasma per $\mathrm{ml}$. of assay sample is low (average, 4.0 per cent; range, 1.6 to 7.0 per cent). This indicated that the values found for these amino acids may represent approximately the concentrations of the free amino acids present in the plasma.

The average mean deviations for arginine (3.9 per cent), methionine ( 3.4 per cent), and threonine ( 5.8 per cent) were low, but their observed concentrations decreased after hydrolysis. This may indicate the presence in unhydrolyzed plasma of a stimulatory substance which could stimulate growth of the test organisms. Comparable decreases of apparent free arginine and serine after hydrolysis have been observed in the livers of tumorous rats (5). The high mean deviations and/or the changes following hydrolysis observed for glycine, isoleucine and serine might be ascribed to the presence of conjugated amino acids or other types of substances stimulatory or inhibitory to the growth of the assay organisms. It is assumed that average values for amino acids calculated from assay data of this character have relative significance when the conditions are rigidly standardized (4). The reliability of the observed concentrations of aspartic and glutamic acids may be questioned since spontaneous (or other) hydrolysis of asparagine and glutamine may contribute to these values (4). It is of interest that Stein and Moore (8) found no change in concentration of arginine and serine and a slight increase for threonine after hydrolysis, while the relative changes for the remaining eleven amino acids were in agreement with the present results. 
Concentration of apparent free amino acids in uremic human plasma and its relation to that found in normal human plasma

It is apparent from the data in Tables II and III that histidine, isoleucine, leucine, methionine, phenylalanine, threonine and valine were significantly decreased in the plasma of Patient No. 1, and that methionine and threonine were decreased in the plasma of all six patients. The decrease of the above amino acids was apparent both in the hydrolyzed and unhydrolyzed state. It is not clear at this time whether the consistent decrease of some amino acids in uremic human plasma reflects a physiological change brought about by the uremic state as such, or whether these low amino acid levels in uremia were secondary to the prolonged administration of the low protein diet which is standard treatment in uremic patients. The overnight fasting of the donors of normal plasma specimens probably did not greatly influence the blood amino acid concentrations of these healthy young men who may have been accustomed to a high protein diet, which may explain the higher levels of many amino acids in their blood. In future experiments of this character it may become necessary to match the patients and donors as to age, hormone and vitamin level, and to induce the donors to submit to the standard uremic dietary regime for a lengthy period prior to the procurement of the plasma specimens. Irrespective of its mechanism, the reduced plasma level of the amino acids mentioned here may well contribute to the poor wound healing, diminished antibody formation and decreased hemoglobin synthesis which suggest inhibition of protein synthesis in the uremic syndrome.

It is apparent from the data in Tables II and III that the concentration of arginine, glycine and proline were markedly higher in uremic plasma than in normal plasma both before and after hydrolysis. The significant increase in apparent arginine has already been noted by Schmidt, McElvain, and Bowen (9) (who concluded, however, that such changes fell within the normal variability of their patients) and also by Dunn, Murphy, and Salisbury (4) and by Doolan, Harper, Watten, Hutchin, and Canada (10) who concluded that the increased concentration of arginine in uremic plasma might well be due to the accumulation of urea, which would retard the enzymatic conversion of arginine into urea and ornithine on the basis of a mass action. Intravenously administered arginine is not very toxic (13) but a chronically elevated arginine concentration may predispose to or cause secondary derangements of protein metabolism which are not observable in acute amino acid infusions. A toxic effect of the chronically elevated plasma arginine level in uremia is therefore not ruled out on the basis of present knowledge.

The data presented here, as well as previous measurements of plasma amino acids in uremic dogs (4), demonstrate the marked elevation of apparent free plasma glycine which occurs in the

TABLE III

Concentration of some protein metabolites in unhydrolyzed human plasma*

\begin{tabular}{|c|c|c|c|c|c|c|c|}
\hline \multirow[b]{2}{*}{ Substance } & \multirow{2}{*}{$\begin{array}{c}\text { Normal } \\
\text { (Pooled, o') }\end{array}$} & \multicolumn{6}{|c|}{ Uremic } \\
\hline & & G. (d) & M. (a) & K. (a) & E. H.t (ন) & C. (0) & S. G. ( 8) \\
\hline 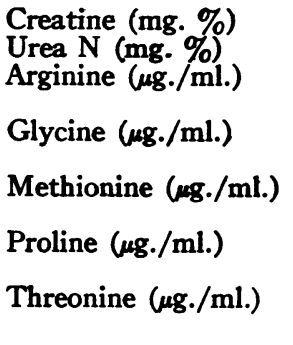 & $\begin{array}{c}4.7 \ddagger \\
3.9 \\
22 \\
11 \\
4.1 \\
3.4 \\
6.6 \\
1.6 \\
19 \\
5.8\end{array}$ & $\begin{array}{r}17 \\
160 \\
18 \\
4.1 \\
52 \\
2.3 \\
2.2 \\
4.2 \\
18 \\
2.3 \\
8.7 \\
6.0\end{array}$ & $\begin{array}{r}18 \\
134 \\
12 \\
8.3 \\
70 \\
6.4 \\
3.6 \\
12 \\
18 \\
2.5 \\
8.7 \\
5.9\end{array}$ & $\begin{array}{r}20 \\
130 \\
14 \\
3.8 \\
122 \\
10 \\
3.8 \\
11 \\
49 \\
1.8 \\
10 \\
12\end{array}$ & $\begin{array}{l}300 \\
3.6 \\
15 \\
80 \\
32 \\
2.0 \\
7.6 \\
9.2 \\
11 \\
9.6 \\
8.1\end{array}$ & $\begin{array}{r}16 \\
200 \\
13 \\
4.4 \\
32 \\
4.0 \\
3.0 \\
10 \\
24 \\
3.5 \\
9.8 \\
9.3\end{array}$ & $\begin{array}{r}17 \\
140 \\
5.4 \\
6.2 \\
102 \\
13 \\
1.7 \\
10 \\
19 \\
4.2 \\
6.5 \\
12\end{array}$ \\
\hline
\end{tabular}

* Data in micrograms per $\mathrm{ml}$.; figures in italics are mean deviations from the mean in per cent.

$\dagger$ Patient E. H. received penicillin and streptomycin four days before procurement of blood specimen.

¥ Amino acid data for normal blood taken from Table II. 
uremic syndrome. De Vries and Alexander (14) had already demonstrated slight elevations of free plasma glycine in five of ten patients with mild uremia and Braun, Kisfaludy, and Dubsky (15) have reported similar findings in two patients. Glycine is known to cause potassium extrusion when added to media containing suspended cells (16), and to cause severe toxic effects when administered intravenously to dogs (17) and humans (18). An investigation of the role of the glycine molecule in uremia appears indicated. - It is not believed that a toxic effect of elevated plasma glycine levels in uremia is due to the increase in free plasma ammonia which has been observed following glycine infusions in dogs and patients (17). The plasma ammonia of uremic patients is not consistently elevated $(19,20)$, and the symptomatology of uremia cannot be reproduced by infusion of ammonia and is not identical with other clinical conditions in which plasma ammonia is increased (21). The elevated plasma glycine content of uremic plasma could be caused by a deficient glycine excretion on the part of the failing kidney, by reduced glycine transformations, or by increased glycine production in the uremic state.

The data presented here also show a distinct elevation of the proline content of uremic plasma. The sources of the excessive plasma proline and its possible contribution to the uremic symptomatology are unknown and await investigation.

In Patient No. 1 the concentration of aspartic acid, glutamic acid, methionine and lysine became markedly increased after hydrolysis while the concentration of other amino acids varied to a lesser degree. It may be inferred from these results that the concentration of asparagine and glutamine is substantially higher in uremic than in normal plasma, an inference which is consistent with the role of these substances as ammonia acceptors. The minor but significant post-hydrolysis variations in the concentration of histidine, lysine, methionine, serine and threonine suggest the existence of microbiologically inactive conjugates of these amino acids in uremic but not in normal deproteinized plasma.

While marked differences existed between the concentrations of individual amino acids in normal and uremic unhydrolyzed plasma filtrates, the net increase of total free amino acids in uremic plasma was only 93 per cent. This would seem to be in agreement with the observation that, although the urea content might increase as much as 3000 per cent, total free amino acid nitrogen in uremic plasma does not increase more than about 100 per cent $(22,23)$. The major portion of the large net difference following hydrolysis can be ascribed to increases of arginine, asparagine, glutamine and proline in uremic plasma. Levenson, Howard, and Rosen (23) noted the presence of a heterogeneous amino acid conjugate which tended to parallel the urea concentration in patients with severe battle wounds and mild uremia who had to be subjected to heroic and multiple therapeutic maneuvers. The principal constituents of this conjugate were usually glutamic acid, glycine, leucine and proline, whereas in normal plasma it was composed of glutamic acid, glycine and threonine.

\section{SUM MARY}

Apparent free plasma amino acids were measured with microbiological methods on a pooled plasma sample of nine normal fasting young men and on individual plasma samples of six patients with severe uremia which was secondary to chronic glomerulonephritis.

The authors' data for the apparent free amino acid levels of normal human plasma are, on the whole, comparable with data for free amino acid content of human plasma reported by other investigators.

In extension of parallel investigations of the free amino acid content of normal and uremic (nephrectomized) dog plasma, it was found that the content of free arginine, glycine and proline was definitely elevated in five of six uremic human plasma specimens. Several other amino acids appear to be significantly decreased in uremic human plasma.

\section{REFERENCES}

1. Bradley, S. E., The Pathologic Physiology of Uremia in Chronic Bright's Disease. Springfield, Thomas, 1948.

2. Merrill, J. P., The Treatment of Renal Failure. New York, Grune and Stratton, 1955.

3. Olsen, N. S., and Bassett, J. W., Blood levels of urea nitrogen, phenol, guanidine and creatinine in uremia. Am. J. Med., 1951, 10, 52.

4. Dunn, M. S., Murphy, E. A., and Salisbury, P. F., Free and apparent amino acids in deproteinized 
plasma of normal and of uremic (nephrectomized) dogs. Proc. Soc. Exper. Biol. \& Med., 1956, 92, 682.

5. Dunn, M. S., and Murphy, E. A., Effect of ethionine alone or with amino acids on tumor growth, carcass weight and nonprotein amino acids in rat liver. Cancer Research, 1955, 15, 760.

6. Dunn, M. S., Camien, M. N., Malin, R. B., Murphy, E. A., and Reiner, P. J., Percentages of twelve amino acids in blood, carcass, heart, kidney, liver, muscle, and skin of eight animals. Calif. Univ. Pub. Physiol., 1949, 8, 293.

7. Hier, S. W., and Bergeim, O., The microbiological determination of certain free amino acids in human and dog plasma. J. Biol. Chem., 1946, 163, 129.

8. Stein, W. H., and Moore, S., The free amino acids of human blood plasma. J. Biol. Chem., 1954, 211, 915.

9. Schmidt, E. G., McElvain, N. F., and Bowen, J. J., Plasma amino acids and ether-soluble phenols in uremia. Am. J. Clin. Path., 1950, 20, 253.

10. Doolan, P. D., Harper, H. A., Watten, R. H., Hutchin, M. E., and Canada, R. O., Changes in plasma amino acids and blood oxygen content during dialysis with the Kolff-Merrill artificial kidney. Trans. Am. Soc. Artificial Internal Organs, 1956, 2, 113.

11. Harper, J. B., Hutchin, M. E., and Kimmel, J. R., Concentration of nineteen amino acids in plasma and urine of fasting normal males. Proc. Soc. Exper. Biol. \& Med., 1953, 80, 768.

12. Huisman, T. H., The concentration of different amino acids in the blood plasma in children suffering from rickets and scurvy. Pediatrics, 1954, 14, 245.

13. Najarian, J. S., and Harper, H. A., Comparative effect of arginine and monosodium glutamate on blood ammonia. Proc. Soc. Exper. Biol. \& Med., 1956, $92,560$.

14. De Vries, A., and Alexander, R., Studies on amino acid metabolism. II. Blood glycine and total amino acids in various pathological conditions, with observations on the effects of intravenously administered glycine. J. Clin. Invest., 1948, 27, 655.

15. Braun, P., Kisfaludy, S., and Dubsky, M., Quantitative analysis of free amino acids in normal and pathological human serum and urine. Acta med. acad. sc. Hung., 1955, 7, 147.

16. Christensen, H. N., and Riggs, T: R., Concentrative uptake of amino acids by the Ehrlich mouse ascites carcinoma cell. J. Biol. Chem., 1952, 194, 57.

17. Harper, H. A., Najarian, J. S., and Silen, W., Effect of intravenously administered amino acids on blood ammonia. Proc. Soc. Exper. Biol. \& Med., 1956, 92, 558.

18. Doolan, P. D., Harper, H. A., Hutchin, M. E., and Alpen, E. L., The renal tubular response to amino acid loading. J. Clin. Invest., 1956, 35, 888.

19. Russell, D. S., Ammonia content of the blood in nephritis. Biochem. J., 1923, 17, 72.

20. Van Slyke, D. D., Linder, G. C., Hiller, A., Leiter, L., and McIntosh, J. F., The excretion of ammonia and titratable acid in nephritis. J. Clin. Invest., 1926, 2, 255.

21. McDermott, W. V., Jr., and Adams, R. D., Episodic stupor associated with an Eck fistula in the human with particular reference to the metabolism of ammonia. J. Clin. Invest., 1954, 33, 1.

22. Berglund, H., Nitrogen retention in chronic interstitial nephritis and its significance. J. A. M. A., 1922, 79, 1375.

23. Levenson, S. M., Howard, J. M., and Rosen, M. A., Studies of the plasma amino acids and amino conjugates in patients with severe battle wounds. Surg., Gynec. \& Obst., 1955, 101, 35. 